\title{
On the difference of H-mode power threshold in divertor and limiter tokamaks
}

\author{
D Kalupin ${ }^{1}$, M Z Tokar ${ }^{1}$, B Unterberg ${ }^{1}$, X Loozen ${ }^{1}$, D Pilipenko ${ }^{2}$, \\ R Zagorski ${ }^{3}$ and TEXTOR Contributors \\ ${ }^{1}$ Institut für Plasmaphysik, Forschungszentrum Jülich GmbH, EURATOM Association, D-52425 \\ Jülich, Germany (partner in Trilateral Euregio Cluster) \\ ${ }^{2}$ Université Libre de Bruxelles, CP 231 Bvd. Du Triomphe, Association EURATOM- Etat Belge, \\ B-1050, Bruxelles, Belgium \\ ${ }^{3}$ Institute of Plasma Physics and Laser Microfusion, EURATOM Association, 00-908 Warsaw, \\ Poland \\ E-mail: d.kalupin@fz-juelich.de
}

Received 27 October 2005

Published 20 April 2006

Online at stacks.iop.org/PPCF/48/A309

\begin{abstract}
The difference in the H-mode power threshold in divertor and limiter configurations is numerically investigated by analysing the effect of boundary conditions imposed on the last closed magnetic surface (LCMS) and given by prescribed density and temperature $e$-folding lengths, $\delta_{n}$ and $\delta_{T}$, respectively. It is demonstrated that the variation of $\delta_{n}$ and $\delta_{T}$ significantly affects the H-mode power threshold. This is explained by the change in the balance between conductive and convective heat losses at the edge. For the ratio $\delta_{n} / \delta_{T}$ large enough, when the convective loss does not exceed $45 \%$ of the total power, the threshold agrees well with the experimental multi-machine scaling for divertor tokamaks. With reduction in $\delta_{n} / \delta_{T}$ and increase in convective loss above this critical level, the power threshold significantly exceeds the scaling, in agreement with observations on different limiter tokamaks. By considering the power and particle balances in the scrape-off layer it is shown that the ratio $\delta_{n} / \delta_{T}$ is controlled by the distance which recycling neutrals pass before entering the confined plasma and which is normally much larger in divertor machines than in the limiter ones. The calculations for the limiter tokamak TEXTOR have predicted the experimentally found conditions for the $\mathrm{L}-\mathrm{H}$ transition in advance.
\end{abstract}

\section{Introduction}

The nature of high confinement mode (H-mode) [1], foreseen as the main operational scenario for ITER [2], remains a subject of intensive experimental and theoretical studies. On the one hand, it is now well established that the transition from low (L) to H-mode occurs due to strong reduction in anomalous transport of particles and energy driven by plasma turbulence. This 
happens in a thin layer near the plasma boundary, called the edge transport barrier (ETB), due to effects of the radial electric field [3], the zonal flows [4] and other mechanisms when, for example, the heating power exceeds a certain threshold. On the other hand, many important questions remain open; in particular, why is this threshold so different in divertor and limiter tokamaks?

The multi-machine scaling, established by analysing experimental data from different devices with divertors [5], predicts that the transition to the H-mode takes place when the loss power, $P_{\mathrm{L}}$, exceeds the threshold value in megawatts:

$$
P_{\text {th }}=0.042 \bar{n}_{\mathrm{e}}^{0.64} B^{0.78} S^{0.94}
$$

where $P_{\mathrm{L}}$ is the sum of the Ohmic power and auxiliary heating power from which the time derivative of the plasma stored energy is subtracted, $\bar{n}_{\mathrm{e}}$ is the line-averaged electron density in $10^{20} \mathrm{~m}^{-3}, S$ the plasma surface area in $\mathrm{m}^{2}$ and $B$ the toroidal magnetic field in Tesla. At the same time, the power required for the $\mathrm{H}$-mode onset in limiter tokamaks is significantly higher than that given by equation (1) [6]. For instance, experiments on the limiter tokamak TUMAN3 and its upgraded version TUMAN-3M have shown that the transition to the H-mode occurs at a power exceeding more than twice that of $P_{\text {th }}$ [7]. In TFTR the required power was three times larger than $P_{\text {th }}[8]$.

In the present paper we focus on the role of boundary conditions imposed on plasma parameters at the LCMS. It is demonstrated that these conditions, strongly related to the behaviour of recycling neutrals, determine the H-mode power threshold through the change in the balance between convective and conductive contributions to the heat losses at the edge, where the strong convective heat losses at the plasma edge prohibit the $\mathrm{L}-\mathrm{H}$ transition. Previously, the effect of neutrals on the L-H transition was discussed particularly in [9], where the bifurcation in the edge plasma parameters by changing the neutral influx through separatrix was examined on the basis of a OD model. In the present contribution we perform a 1D consideration of the entire confined plasma region. This allows, in particular, distinguishing the $e$-folding lengths of the plasma parameters averaged over the edge region and those at the separatrix. The latter ones cannot be determined in the framework of a OD model for the edge but are of much importance, as well as the neutral influx, for the $\mathrm{L}-\mathrm{H}$ transition. In order to assess these parameters we also consider the transport processes in the scrape-off layer which where disregarded in [9]. In addition, besides ITG and resistive ballooning modes our transport model includes unstable drift Alfven waves, which as is believed now are the main cause of the edge anomalous transport in the L-mode [10-12]. At the same time, our approach does not consider yet the effect of the Reynolds stress on turbulence suppression, playing the dominant role in [9]. This effect will be taken into account in our future studies.

Note that another possible explanation of the strong difference in L-H transition threshold power was suggested in [13] and refers to distinctive difference in magnetic shear behaviour near the edge in limiter and divertor tokamaks. This effect of magnetic shear is included in the transport model used in our calculations. Nevertheless, the results presented here demonstrate that even with a weak shear at the plasma edge, corresponding to the limiter configuration, one can achieve the H-mode power threshold in agreement with the multi-machine scaling if strong convective heat losses arising due to ionization of recycling neutrals are prohibited.

The rest of the paper is organized as follows. In section 2 the RITM code is briefly described: results of computations showing the influence of the boundary conditions on the ETB formation and $\mathrm{H}$-mode power threshold are presented. In section 3 the improved two-point model for the scrape-off layer is used to analyse the variation of logarithmic decay lengths at the LCMS with the distance travelled by neutrals in the SOL. In section 4 the results of preparatory 
modelling on the H-mode power threshold are compared with the first experimental data on the ETB formation in the limiter tokamak TEXTOR. In section 5 the conclusions of the paper are given.

\section{Influence of the boundary conditions on the ETB formation}

To study the formation of ETB the 1.5D transport code, RITM, has been used. The transport equations solved by the RITM code and the code structure are described in detail in $[10,14,15]$. The code allows modelling of the radial profiles for diverse plasma parameters over the whole minor radius, from the plasma axis to the LCMS. RITM solves 1D transport equations for the densities and temperatures of electrons, main and impurity ions and the current diffusion equation. The sources of main plasma particle are computed by taking into account the ionization of main neutrals recycling from limiters or divertor plates into the confined volume, from neutral beam injection and impurities eroded or puffed into the plasma. The code allows considering simultaneously all charged states of impurities such as $\mathrm{He}, \mathrm{C}, \mathrm{O}, \mathrm{Ne}, \mathrm{Si}$ and Ar. The heat sources due to Ohmic and auxiliary heating and energy exchange between different plasma components by elastic and non-elastic collisions are taken into account. The particle fluxes include diffusive and convective components. The heat fluxes are composed of conductive and convective contributions associated with the temperature gradient and particle flow, respectively. In particular, the latter can dominate the heat losses at the plasma edge in a limiter configuration, where the plasma column is placed much closer to the plasma-facing components than in divertor machines. For a given heating power, a larger convection fraction results in lower temperature and its gradient. Thus, the plasma collisionality increases and the pressure gradient reduces. It was first demonstrated in [11] and reproduced afterwards by calculations with RITM [10] that increasing collisionality and decreasing pressure gradient intensify the DA-instability dominating the turbulent transport at the edge under L-mode conditions. Therefore, the L-H power threshold is expected to be higher in limiter devices where the heat losses at the edge are dominated by the convection.

The switch between conductive and convective heat losses can be involved in RITM modelling through change in the boundary conditions at the LCMS, $r=a$ :

$$
\nabla n=-n / \delta_{n}, \nabla T=-T / \delta_{T} .
$$

Here $\delta_{n}$ and $\delta_{T}$ are the $e$-folding lengths of the plasma density and temperature, respectively, being prescribed in RITM calculations. Indeed, the fraction of convective losses, $-3 T D_{\perp} \nabla n$, in the total one, $-3 T D_{\perp} \nabla n-\chi_{\perp} n \nabla T$, is given at the LCMS by the value $\xi_{\text {conv }}=$ $\left(1+\left(\chi_{\perp} / 3 D_{\perp}\right)\left(\delta_{n} / \delta_{T}\right)\right)^{-1}$. By varying the ratio $\delta_{n} / \delta_{T}$, this can be changed from 0 to 1 equivalent to the transition from the pure conductive to the pure convective regime. In the next section we show that the ratio $\delta_{n} / \delta_{T}$ and, therefore, the fraction $\xi_{\text {conv }}$ at the plasma edge are controlled by the penetration of the recycling neutrals. The parameter which determines the penetration of neutrals through the SOL is the ratio of the distance between the neutralizing plates and the confined plasma to the neutral mean free path. This parameter increases in the switch from limiter to divertor configuration due to the larger distance which should be travelled by neutrals.

Figure 1 displays the plasma pressure averaged over normalized minor radii between $0.95<\rho<0.98$, where the most significant change during transition to H-mode occurs, and the fraction of convective heat losses at the LCMS versus $\delta_{n}$ and $\delta_{T}$, found in RITM calculations for TEXTOR with $B=1.9 \mathrm{~T}, \bar{n}_{\mathrm{e}}=2 \times 10^{19} \mathrm{~m}^{-3}$ and total heating of $2 \mathrm{MW}$. In computations where $\delta_{T}$ was fixed and $\delta_{n}$ varied from 0.5 to $5 \mathrm{~cm}$, presented in figure $1(a)$, the strong increase in the averaged edge pressure, caused by the reduction in the edge transport, is found if the 

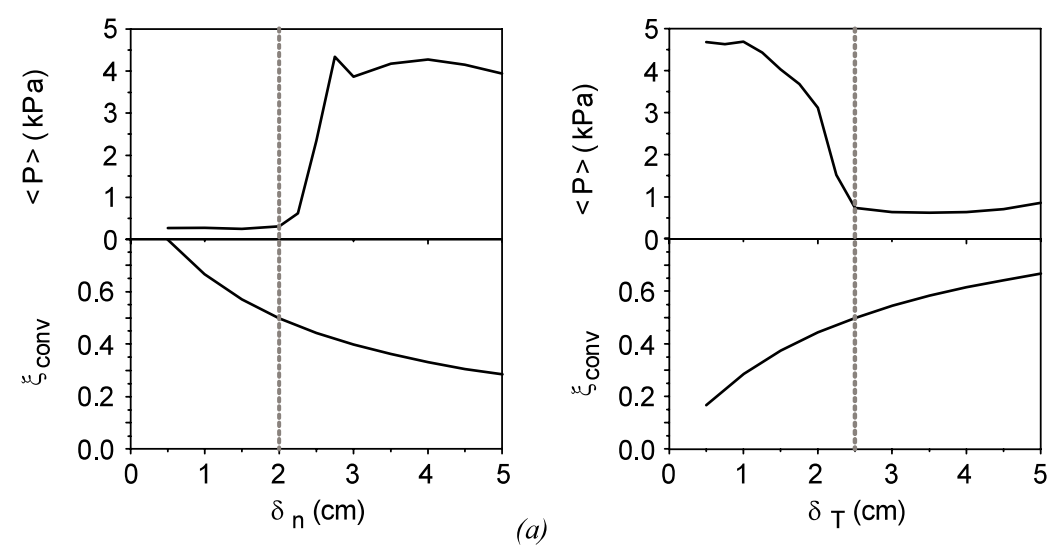

Figure 1. Edge plasma pressure and fraction of convective heat losses at the LCMS versus the density $(a)$ and the temperature $(b) e$-folding lengths.
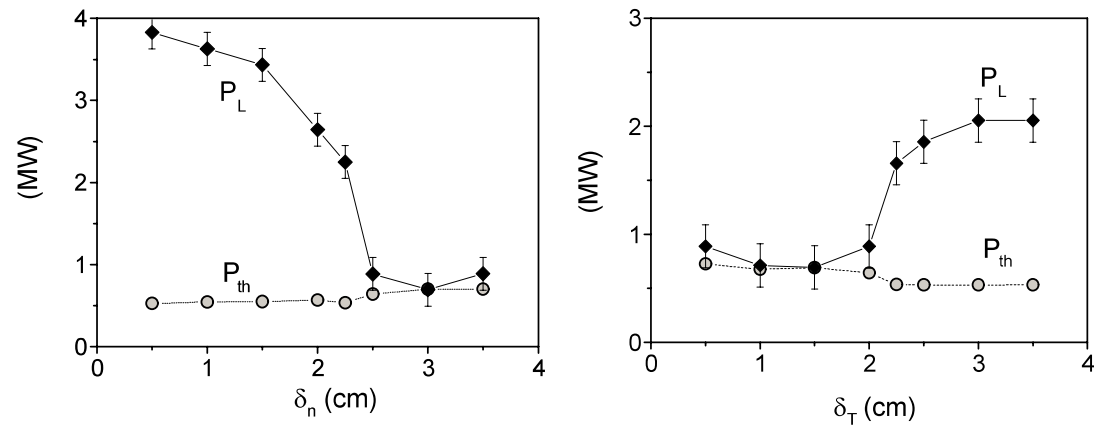

Figure 2. H-mode threshold in TEXTOR computed with RITM $(\diamond)$ and according to multi-machine scaling, equation (1), (O) versus the density (left) and the temperature (right) $e$-folding lengths at the LCMS.

density decay length is larger than a certain value. On the contrary, the computations done for the fixed $\delta_{n}$ and with different values of $\delta_{T}$ show the same pressure increase if $\delta_{T}$ is lower than a certain limit, figure $1(b)$. In both the cases the transition from the state with a high transport and low confinement to the regime with ETB, leading to the development of the pedestal on the pressure profile, occurs when the fraction of convective losses is below 45-50\%. A smaller convection fraction requires larger temperature and its gradient. Correspondingly, the plasma collisionality decreases and the pressure gradient grows resulting in a decreasing anomalous transport: DA waves are destabilized by collisions but stabilized by the radial pressure gradient which has a component along perturbed magnetic field lines acting against the friction force caused by collisions (see equation (13) in [10]). When DA transport contribution is reduced to a certain critical level, the bifurcation into the H-mode occurs (see [16]).

The variation with $\delta_{n}$ of the power threshold for the ETB onset computed with RITM for $\delta_{T}=2 \mathrm{~cm}$ is shown in figure 2(a). At low $\delta_{n}$, when the heat loss is dominated by the convection, the computed critical power significantly exceeds predictions of the multi-machine scaling, (equation 1). If the density $e$-folding length increases and the fraction of convective heat loss reduces, the computed critical power, $P_{\mathrm{L}}$, drops and comes, for $\delta_{n}>2.5 \mathrm{~cm}$, very close to that predicted by equation (1). A similar behaviour is seen when $\delta_{n}$ is fixed, at $2.5 \mathrm{~cm}$, and 


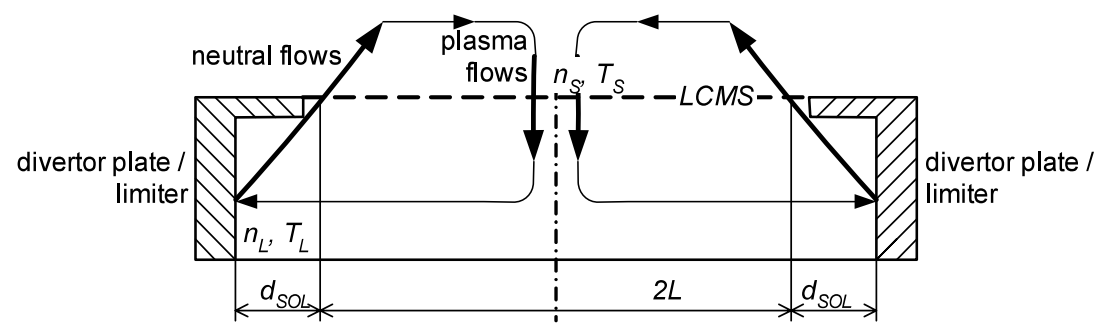

Figure 3. Schematic view of particle recycling at the plasma edge in a tokamak.

$\delta_{T}$ is varied, figure $2(b)$. In both cases the multi-machine scaling is well reproduced when the fraction of convective heat losses is smaller than a characteristic level of $50 \%$.

\section{Effect of the distance between LCMS and neutralizing plates}

The difference between divertor and limiter configurations can be characterized by the different ratio of the distance $d_{\mathrm{SOL}}$, which recycling neutrals pass in the SOL before they escape into the confined volume of their mean free path. In the divertor case $d_{\mathrm{SOL}}$ is normally much larger than in the limiter one. This results in a different ordering of the $e$-folding lengths for the density and temperature at the LCMS, $\delta_{n}$ and $\delta_{T}$, respectively. In order to demonstrate this we apply an improved two-point model for the SOL [17], which takes into account the ionization of recycling neutrals and convective heat transport.

Consider a schematic picture of particle flows at the plasma edge, (figure 3). Charged particles diffuse through the LCMS out of the confined region into the SOL. Here they move along the field lines towards the neutralizing plates where electrons and ions recombine into neutral particles. The latter recycle back into the SOL plasma and partly penetrate into the confined volume. Here neutrals are ionized and generated charged particles spread over the magnetic surfaces. In the following consideration we assume that the plasma density at the symmetry point along the length of the LCMS from one neutralizing plate to the other, $n_{\mathrm{s}}$, and the heating power transferred into the SOL, $P_{\text {heat }}$, being the total perpendicular heat flux from the confined plasma multiplied by area of the LCMS, are fixed.

We assume that the energy losses in the main part of the SOL, e.g. charge-exchange, are small [18]. Therefore, only two main channels for the energy loss are remaining: (i) $P_{\text {heat }}$ is partly transmitted to the neutralizing plates by the stream of charged particles and (ii) it is partly spent on the ionization of recycling neutrals. The particle flux reaching the plate is given by the product of the density of charged particles at the plate, $n_{\mathrm{L}}$, and ion sound speed acquired by particles at the plate, $V_{s}=\sqrt{2 T_{\mathrm{L}} / m_{\mathrm{i}}}$. Moreover, since only the poloidal balance is considered, this product should be multiplied by $\sin \psi$, where $\psi$ is the pitch angle between the magnetic field and the toroidal direction. Assuming, $E_{\mathrm{i}}$ is the energy spent on the ionization of a neutral, including its excitation, and $\gamma T_{\mathrm{L}}$ is the energy transmitted to the plate, where $T_{\mathrm{L}}$ is the temperature at the plate and $\gamma$ is the energy transmission factor, one gets

$$
P_{\text {heat }}=4 \pi R \delta\left(\gamma T_{\mathrm{L}}+E_{\mathrm{i}}\right) n_{\mathrm{L}} V_{s} \sin \psi .
$$

Here $R$ is the major radius and $\delta$ the effective radial thickness of the SOL.

The balance of particles in the SOL represents the equality of the charged particles influx from the confined plasma, $\Gamma_{\mathrm{LCMS}}$, and the outflow of recycling neutrals through the LCMS:

$$
\Gamma_{\mathrm{LCMS}}=4 \pi R \delta n_{\mathrm{L}} V_{s} \sin \psi \exp \left(-n_{\mathrm{L}} \sigma_{*} d_{\mathrm{SOL}}\right) .
$$




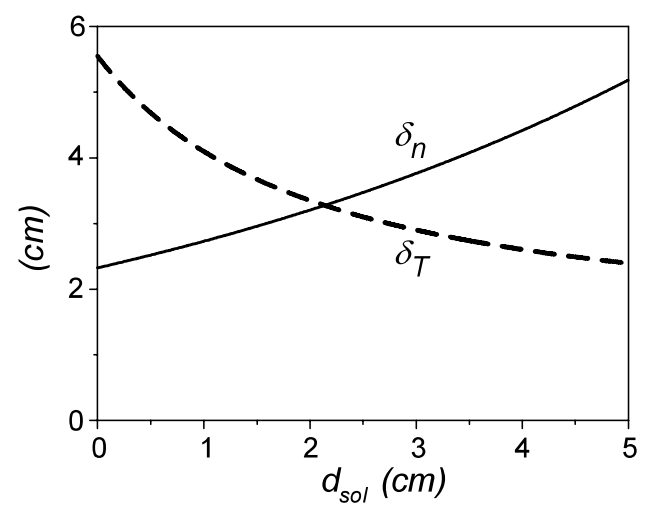

Figure 4. Density and temperature e-folding lengths at the LCMS versus the distance to the neutralizing plates.

The difference between divertor and limiter geometry enters into this equation though the exponential factor taking into account the ionization of neutrals in the SOL, where $n_{\mathrm{L}} \sigma_{*} d_{\mathrm{SOL}}$ is the ratio of the distance travelled by neutrals in the SOL to their mean free path, with $\sigma_{*}$ being the effective cross-section for the neutral losses due to ionization and charge-exchange.

The plasma parameters near the plate and at the LCMS are related through the conservation of the total parallel momentum [17]:

$$
2 n_{\mathrm{L}} T_{\mathrm{L}}=n_{\mathrm{S}} T_{\mathrm{S}},
$$

and the integral of the heat transport equation with conductive and convective energy transfer included [18]:

$$
\begin{aligned}
& \frac{T_{\mathrm{C}}^{5 / 2}}{2}\left[\ln \frac{\sqrt{T_{\mathrm{C}}}+\sqrt{T_{\mathrm{S}}}}{\sqrt{T_{\mathrm{C}}}-\sqrt{T_{\mathrm{S}}}}-\ln \frac{\sqrt{T_{\mathrm{C}}}+\sqrt{T_{\mathrm{L}}}}{\sqrt{T_{\mathrm{C}}}-\sqrt{T_{\mathrm{L}}}}\right]=\frac{T_{\mathrm{S}}^{5 / 2}-T_{\mathrm{L}}^{5 / 2}}{5}+T_{\mathrm{C}} \frac{T_{\mathrm{S}}^{3 / 2}-T_{\mathrm{L}}^{3 / 2}}{3} \\
& +T_{\mathrm{C}}^{2}\left(T_{\mathrm{S}}^{1 / 2}-T_{\mathrm{L}}^{1 / 2}\right)+\frac{5 L^{2}}{A_{k} \delta} \frac{\Gamma_{L C M S}}{S}
\end{aligned}
$$

where $T_{\mathrm{s}}$ is the temperature at the symmetry point along the LCMS and $T_{\mathrm{C}}=5 P_{\text {heat }} / \Gamma_{\mathrm{LCMS}}$ the maximum level of $T_{\mathrm{s}}$ approached in the case when $P_{\text {heat }}$ is transported by the particle flow only and no parallel conduction is involved; the latter is given by Spitzer formula, $\kappa_{\|}=A_{k} T^{5 / 2}$.

Applying the boundary conditions at the LCMS equations (2), we get

$$
\begin{aligned}
& \frac{\Gamma_{\mathrm{LCMS}}}{S}=\frac{D_{\perp}}{\delta_{n}} n_{S} . \\
& \frac{P_{\text {heat }}}{S}=\left(\frac{3 D_{\perp}}{\delta_{n}}+\frac{\chi_{\perp}}{\delta_{T}}\right) n_{S} T_{\mathrm{S}} .
\end{aligned}
$$

In order to close the set of equations for the determination of parameters $T_{\mathrm{s}}, T_{\mathrm{L}}, n_{\mathrm{L}}, \Gamma_{\mathrm{LCMS}}$, $\delta, \delta_{n}$ and $\delta_{T}$ for given $n_{s}$ and $P_{\text {heat }}$, we assume the relation

$$
\delta=\delta_{n} \delta_{T} /\left(\delta_{n}+\delta_{T}\right),
$$

which suggests that the SOL width does not exceed the $e$-folding lengths of the density and the temperature at the LCMS. Figure 4 demonstrates the variation of $\delta_{n}$ and $\delta_{T}$ with $d_{\mathrm{SOL}}$ computed for TEXTOR parameters with $n_{s}=2 * 10^{13} \mathrm{~cm}^{-3}$ and $P_{\text {heat }}=2 \mathrm{MW}$ by solving equations (3)-(9) numerically. For small $d_{\mathrm{SOL}}$ recycling neutrals escape freely into the confined volume and provide a large source of charged particles there. In order to transport the generated particle flux back into the SOL with diffusion, a large radial density gradient should develop 


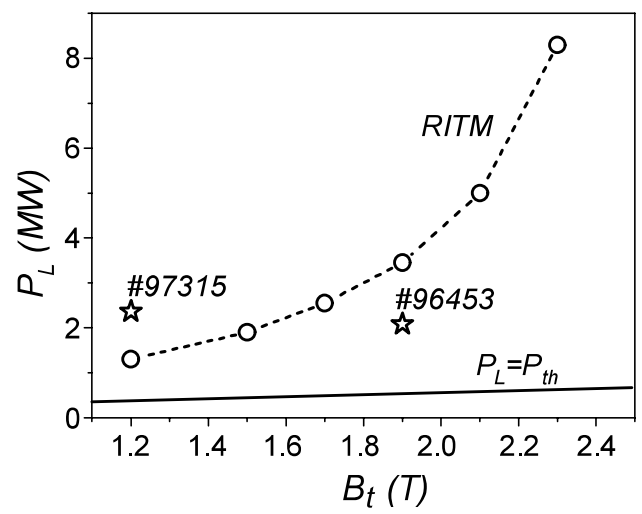

Figure 5. Threshold power for $\mathrm{L}-\mathrm{H}$ transition computed with RITM $(\mathrm{O})$ and according to multimachine scaling, equation (1)(- $)$.

and this results in a small $\delta_{n}$. Because of large convective heat losses the heat conduction cannot contribute a lot to the total heat flux; the temperature gradient is relatively small and $\delta_{T}$ is large. For large $d_{\mathrm{SOL}}$, the neutral attenuation in the SOL is high, the particle flux through the LCMS is weak and $\delta_{n}$ is large. The heat flux is transported through the LCMS predominantly by conduction, and $\delta_{T}$ is small. Reduced convective energy losses make the transition to the improved confinement state easier. This offers an explanation for the experimentally observed difference between the H-mode power thresholds in limiter and divertor configurations.

\section{Predictions for the ETB onset in TEXTOR}

Calculations with the RITM code have been done in order to predict under what conditions the $\mathrm{H}$-mode can be established in the limiter tokamak TEXTOR. Figure 5 shows the L-H threshold power computed for experimental conditions with $\bar{n}_{\mathrm{e}} \approx 2 * 10^{13} \mathrm{~cm}^{-3}, q_{\text {edge }} \approx 3, \delta_{n}=1 \mathrm{~cm}$ and $\delta_{T}=1.5 \mathrm{~cm}$ versus the toroidal magnetic field. Because of $\delta_{n} / \delta_{T}<1$, a significant fraction of the heat losses through the LCMS is due to particle convection and the threshold significantly exceeds the level predicted by the multi-machine scaling, (equations (1)). The strong dependence of the computed threshold power on the toroidal field is explained as follows. The stabilizing effect of the radial pressure gradient on DA instability is proportional to the ratio of the radial and toroidal components of the magnetic field, (see equations (13) in [10]). The former one is inversely proportional to the perpendicular wave vector of perturbations which is of the order of $1 / \rho_{s}$, where $\rho_{s}$ is the ion Larmor radius. Thus, the stabilizing term varies as $1 / B_{\mathrm{t}}^{2}$. and, therefore, the power threshold strongly reduces with decreasing $B_{\mathrm{t}}$.

Stars in figure 5 represent conditions for the two shots in TEXTOR. In shot \#96453, in spite of the fact that equations (1) predicts the $\mathrm{H}$-mode conditions, the plasma is in the L-mode. This is, however, in agreement with RITM calculation providing for these conditions $P_{\mathrm{L}}>3.5 \mathrm{MW}$. Due to limitations in the heating power from NBI and ICRH, the magnetic field was reduced to $1.2 \mathrm{~T}$ in shot \#97315 in order to achieve improved confinement. When the heating power was increased up to $2.3 \mathrm{MW}$, a sudden reduction in $\mathrm{H}_{\alpha}$ signal accompanied with the increase in plasma gradients at the edge was observed. The time traces of different signals in shots \#96453 and \#97315 are shown in figures 6(a) and $(b)$ respectively. In the shot with the higher magnetic field figure $6(a)$ the plasma stays permanently in the L-mode and its behaviour is very stationary. But, in the shot with the reduced magnetic field (figure $6(b)$ ), some of the signatures 

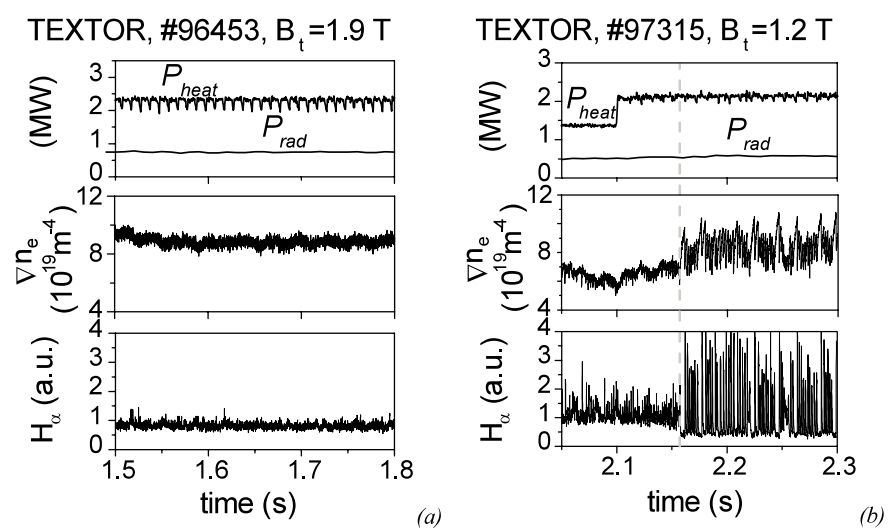

Figure 6. Time traces of the heating and radiated power, density gradient at the plasma edge and $H_{\alpha}$ signal in TEXTOR shots with different magnitudes of the toroidal magnetic field.

characteristic for $\mathrm{L}-\mathrm{H}$ transition were observed at the time $0.06 \mathrm{~s}$ after the full NBI heating was applied. The averaged level of $\mathrm{H}_{\alpha}$-signal drops, which is followed by the appearance of strong bursts on this signal. As MHD analysis is still ongoing, it is not possible to conclude about the relation between these bursts and the MHD activity. Therefore, it is too early to claim that the observed $\mathrm{H}_{\alpha}$-spikes are identical to conventional ELMs. The other observation consistent with what is typically observed during $\mathrm{L}-\mathrm{H}$ transition is the steeping in the density profile at the edge. The density gradient, defined as the difference in the density value measured by two outer most HCN channels divided by the radial distance between channels, increases by 15-20\% at the same time when $\mathrm{H}_{\alpha}$-signal drops. It is important to stress that RITM calculations have predicted in advance the conditions at which the first indications of $\mathrm{L}$ to $\mathrm{H}$ mode transition were observed.

\section{Conclusions}

Calculations done with 1.5D transport code, RITM, show the strong dependence of the L-H threshold power on the boundary conditions at the LCMS. For the given heating power, the transition occurs if the $e$-folding length of density is increased and the temperature $e$-folding length is reduced. This is explained by the transition from the case where heat losses from the confined plasma are mostly due to charged particle convection to the situation with heat losses dominated by the conduction.

The analysis done with two-point model demonstrated that the $e$-folding lengths of plasma parameters at the LCMS and relative contributions of convective and conductive heat losses are determined by the transport of neutrals recycled from neutralizing plates and, in particular, by the ratio of the distance which they should pass in the SOL before entering the confined plasma of their mean free path. The increase in this distance between neutralizing plates and confined plasma, which occurs in the switch from limiter to divertor tokamak, leads to a higher attenuation of the recycling neutral flux due to ionization in the SOL and, therefore, changes the heat flux balance to the conduction-dominated one.

Our computations show that, in plasmas where the energy losses are dominated by the charge particle convection, e.g. in limiter configuration, the heating power required to establish the ETB is up to a few megawatts larger than the value predicted by the inter-machine scaling established for divertor devices. Whereas, in the case of a more closed configuration with the 
heat transport dominated by conduction, the computed threshold power practically coincides with the prediction by this scaling.

Recent observations from the limiter tokamak TEXTOR support the results obtained with the modelling performed prior to the experiment.

\section{References}

[1] ASDEX team 1989 Nucl. Fusion 291959

[2] Shimada M et al 2004 Nucl. Fusion 44350

[3] Terry P W 2000 Rev. Mod. Phys. 72109

[4] Diamond P H et al 1994 Phys. Rev. Lett. 722565

[5] Snipes J A et al 2002 Proc. 19th Int. Conf. on Fusion Energy 2002 (Lyon, France, 2002) (Vienna: IAEA) CD-ROM file TERCT-P/04 and http://www.iaea.org/programmes/ripc/physics/fec2002/html/fec2002.htm

[6] ITPA H-mode Threshold Database Working Group 2004 Proc. 20th Int. Fusion Energy Conf. (Vilamoura, Portugal, 2004) IT/P3-35

[7] Lebedev S V et al 1996 Plasma Phys. Control. Fusion 381103

[8] Bush C E et al 1995 Phys. Plasmas 22366

[9] Carreras B A et al 1996 Phys. Plasmas 34106

[10] Kalupin D et al 2005 Nucl. Fusion 45468

[11] Kerner W et al 1998 Contrib. Plasma Phys. 38118

[12] Janeschitz G et al 1999 J. Nucl. Mater. 266-269 843

[13] Toi K et al 1990 Phys. Rev. Lett 641895

[14] Tokar M Z 1994 Plasma Phys. and Control. Fusion 361819

[15] Kalupin D et al 2001 Plasma Phys. Control. Fusion 43945

[16] Kalupin D et al 2006 Phys. Plasmas 13032504

[17] Stangeby P C 2000 The Plasma Boundary of Magnetic Fusion Devices (Bristol: Institute of Physics Publishing) p 221

[18] Tokar M Z et al 2004 Phys. Plasmas 114610 